\title{
Ongoing outbreak of an acute muscular Sarcocystis-like illness among travellers returning from Tioman Island, Malaysia, 2011-2012
}

D H Esposito (desposito@geosentinel.org) ${ }^{1}$, D 0 Freedman ${ }^{2}$, A Neumayr ${ }^{3}$, P Parola ${ }^{4}$

1. Division of Global Migration and Quarantine, National Center for Emerging and Zoonotic Infectious Diseases, Centers for Disease Control and Prevention, Atlanta, Georgia, United States

2. GeoSentinel Program Office, Division of Infectious Diseases, University of Alabama at Birmingham, Birmingham, Alabama, United States

3. TropNet Coordinating Center, Swiss Tropical and Public Health Institute, Basel, Switzerland

4. EuroTravNet Coordinating Center, University Hospital Institute for Infectious and Tropical Diseases, Aix-Marseille University and Assisitance-Publique Hôpitaux de Marseille, Marseille, France

Citation style for this article:

Esposito DH, Freedman DO, Neumayr A, Parola P. Ongoing outbreak of an acute muscular Sarcocystis-like illness among travellers returning from Tioman Island, Malaysia, 2011-2012. Euro Surveill. 2012;17(45):pii=20310. Available online: http://www.eurosurveillance.org/ViewArticle.aspx?Articleld=20310

As of 4 November, 2012, 100 patients with an acute muscular Sarcocystis-like illness associated with travel to Tioman Island, Malaysia, have been identified. Thirtyfive travelled there mostly during July and August 2011 and 65 mostly during July and August 2012, suggesting an ongoing outbreak. Epidemiological investigations are ongoing. Public health agencies and practicing clinicians should be aware of this rarely-reported disease in humans and consider it as differential diagnosis in travellers returning from Tioman Island.

From 27 July to 4 November 2012, GeoSentinel [1], working with EuroTravNet [2] and TropNet [3], has identified 65 patients with an acute muscular Sarcocystislike illness after recent travel to Tioman Island. All of these patients had traveled to Tioman, located off the east coast of peninsular Malaysia, mostly during July and August, 2012. Reports originated from Germany $(n=25)$, France $(n=20)$, the Netherlands $(n=12)$, Switzerland $(n=3)$, Belgium $(n=2)$, Spain $(n=2)$, and Singapore $(n=1)$. These patients appear to represent the second wave of an outbreak that started in 2011 [4]. An epidemiologic investigation, initiated in November 2011, is ongoing.

\section{Outbreak description}

Starting from October 25, 2011, 35 patients with an acute muscular Sarcocystis-like illness were identified by early 2012; all traveled to Tioman Island, mostly during July and August of 2011. Following months without reports, new patients have been identified since late July 2012, and 65 have been reported to GeoSentinel by 4 November 2012. Cases presented here with limited, preliminary data, are patients reported to GeoSentinel with suspected acute muscular sarcocystosis, defined as an acute illness characterised by prominent musculoskeletal complaints with or without fever, with unexplained eosinophilia and recent travel to Tioman Island.
Although data collection is incomplete, the clinical presentation appears to be identical to that seen last year: almost all patients have experienced fever and myalgia while fewer have complained of arthralgia, asthenia, headache, cough, and diarrhoea. Only some had pruritic rash or edema of the face or an extremity. At least four patients were hospitalised.

Laboratory investigations showed that in many cases, at the onset of symptoms absolute eosinophil counts and serum creatinine phosphokinase (CPK) levels can be normal but begin to be moderately elevated approximately 30 days or more after departure from Tioman Island. Maximum absolute eosinophil counts and serum CPK levels from patients with data available, have typically been $<1,000$ cells/mm3 (norm: $<500$ ) and 11,500 $\mathrm{U} / \mathrm{L}$ (norm: $<200$ ), respectively, but higher values were seen in some patients. To date, all patients with known testing (2011 $n=34 ; 2012 n=8$ ) have been serologically negative for trichinellosis. Eight patients are known to have had a muscle biopsy; for six patients results are available and all revealed myositis (defined as myalgia and inflammation and myocyte degeneration on histopathologic examination of muscle tissue) and in two intramuscular cysts (sarcocysts) were identified.

Among the 65 patients, two asymptomatic persons were identified who, when tested, had elevated eosinophil counts and serum CPK levels. One of them was a child who travelled with several family members, all of whom experienced an acute muscular Sarcocystis-like illness. The other person traveled with a companion who developed an acute muscular Sarcocystis-like illness and whose muscle biopsy showed inflammation but no intramuscular sarcocysts. The initially asymptomatic person subsequently developed myositis and mild myocarditis and was hospitalised. 


\section{Background}

Sarcocystis species are intracellular protozoan parasites with an obligatory two-host predator-prey (definitive-intermediate) lifecycle [5]. Humans are the definitive host for Sarcocystis hominis and Sarcocystis suihominis, which are acquired by eating undercooked sarcocyst-containing beef or pork, respectively. The parasite reproduces sexually in the human intestine, where infection can cause acute gastroenteritis; however, most $S$. hominis and $S$. suihominis infections are likely asymptomatic [6]. Although the specific species have never been identified, humans can become accidental intermediate hosts for one or more of the 130 known Sarcocystis species through ingesting oocysts or sporocysts in food or water contaminated with feces from an infected predator animal. Nonspecific symptoms might arise during asexual reproductive and migratory phases of the parasite within the vascular endothelium and this generalised vasculitis has associated with myositis, myocarditis, and perivascular inflammation of the liver, kidneys, and lungs in experimentally and naturally infected animals [7]. The parasite ultimately disseminates to skeletal and cardiac muscle and forms sarcocysts containing large numbers of parasites that, when mature, are infectious for a definitive host. This phase in the lifecycle may or may not be associated with myocyte degeneration and active inflammation, depending on the specific Sarcocystis species-host species interaction [7]. Definitive diagnosis of muscular sarcocystosis relies on identifying sarcocysts in a muscle biopsy sample; DNA amplification and PCR testing of muscle tissue and serology is experimental and not widely available. No proven treatment exists for human sarcocystosis.

Fewer than 100 cases of human muscular sarcocystosis have been reported in the literature, with most discovered incidentally in asymptomatic persons [6]. Human sarcocystosis is prevalent in Malaysia; a seroprevalence study found evidence of infection in $20 \%$ of 243 Malaysians [8] which is concordant with a $21 \%$ prevalence of sarcocysts found in muscle tissue from 100 consecutive autopsies in Malaysia [9]. The largest reported outbreak of apparent acute muscular disease in the past, affected seven of fifteen United States (US) servicemen on maneuvers in a Malaysian jungle [10]. All but one of them had symptoms, four had myositis and one was confirmed to have sarcocysts in his muscle.

\section{Epidemiological investigation}

GeoSentinel is coordinating an epidemiological investigation to systematically gather detailed demographic, travel, exposure, and clinical data using structured data collection instruments. Case finding activities have identified 100 persons with suspected Sarcocystis species after visiting Tioman Island, mostly during the 2011 and 2012 northern hemisphere summer tourist seasons.

A Sarcocystis-specific Western-blot serological assay is under development at the US Centers for Disease
Control and Prevention (CDC) to assist in differentiating infected from non-infected persons associated with this outbreak. Histologic examination and DNA amplification is being performed at CDC on existing muscle biopsy specimens from outbreak patients to confirm the diagnosis of muscular sarcocystosis and to identify the responsible Sarcocystis species. Updates on the investigation are being provided to public health authorities in Malaysia and countries where patients have been identified.

\section{Discussion and conclusion}

This is the largest reported cluster of patients with suspected acute muscular sarcocystosis. With little known about this disease in humans, practicing clinicians' awareness is likely low and many more as yet unrecognised patients may exist. Moreover, in agreement with a previously reported outbreak [10], our findings indicate that asymptomatic infection, and even late presentation, may occur. Since asymptomatic infection may be possible, the true number of persons infected by Sarcocystis species while visiting Tioman Island could be underestimated.

So far the data are still incomplete however, most patients analysed experienced acute illness characterised by fever and myositis. Myositis with significant eosinophilia but no serologic evidence of trichinellosis is an unusual clinical entity. Given the consistent pattern of illness among patients identified, the universal history of travel to a single small island in south-east Asia, and the presence of sarcocysts in the muscle biopsies of some of the patients, a Sarcocystis species infection with transmission occurring on Tioman Island seems to be the most likely cause of this outbreak. The source of infection for international travellers to Tioman Island remains unknown, but a seasonal pattern of transmission is apparent. This seasonality may reflect peak periods of summer tourist travel to the island, but may also correlate with true seasonallyoccurring contamination of food, water, or the environment, perhaps related to specific weather patterns, seasonal food sources, or other seasonal events.

The epidemiology of human muscular sarcocystosis in Malaysia and neighbouring countries is poorly investigated. Detailed ascertainment of risk behaviours and exposures associated with the current cluster of known cases is included in the survey instrument used for the investigation of the outbreak.

Travellers to Tioman Island should be made aware of this potential travel health risk and be reminded about the necessity to practice safe food and water consumption and proper hygiene. Public health officers and practicing clinicians who are aware of persons with prominent musculoskeletal complaints with or without fever, with unexplained eosinophilia and recent travel to Tioman Island, Malaysia, are encouraged to consider sarcocystosis as differential diagnosis. Moreover, they are invited to participate in our investigation and 
contact the corresponding author to report patients or receive guidance on how to proceed. Data collection forms and information on how to submit clinical samples (serum for Sarcocystis-specific serology and/or tissue for histopathology and Sarcocystis-specific PCR testing) are readily available.

\section{Acknowledgments}

We are grateful to all clinicians and members of the EuroTravNet/GeoSentinel and TropNet networks who contributed to this alert by providing early reports of their patients. EuroTravNet (http://www.eurotravnet.eu) has been funded through the European Centre for Disease Prevention and Control's public tenders 0J/2008/07/08-PROC/2008/019 and $0 J / 2010 / 03 / 16-P R O C / 2010 / 011$ and has been supported by Cooperative Agreement $5 \mathrm{U}_{50} \mathrm{CK}$ o00189from the United States Centers for Disease Control and Prevention. It has been created by grouping the European surveillance sites and network members of GeoSentinel (http://www.geosentinel.org), the global surveillance program of the International Society of Travel Medicine. TropNet (http://www.tropnet. net) is a European network for tropical medicine and travel health.

We also thank the US Centers for Disease Control and Prevention's Parasitic Diseases Branch Reference Laboratory and Infectious Diseases Pathology Branch and to the US Department of Agriculture Agricultural Research Services for diagnostic expertise and support.

The findings and conclusions in this report are the findings and conclusions of the authors and do not necessarily represent the views of the US Centers for Disease Control and Prevention.

\section{References}

1. Freedman DO, Weld LH, Kozarsky PE, Fisk T, Robins R von Sonnenburg F, et al. Spectrum of disease and relation to place of exposure among ill returned travelers. N Engl J Med. 2006;354(2):119-30.

2. Gautret $P$, Cramer JP, Field V, Caumes E, Jensenius $M$, Gkrania-Klotsas E, et al. Infectious diseases among travellers and migrants in Europe, EuroTravNet 2010. Euro Surveill. 2012;17(26): pii=20205. Available from: http://www. eurosurveillance.org/ViewArticle.aspx?Articleld=20205

3. Bouchaud O, Mühlberger N, Parola P, Calleri G, Matteelli A, Peyerl-Hoffmann G, et al. Therapy of uncomplicated falciparum malaria in Europe: MALTHER - a prospective observational multicentre study. Malar J. 2012;11:212.

4. Centers for Disease Control and Prevention (CDC). Notes from the Field: acute muscular sarcocystosis among returning travelers - Tioman Island, Malaysia, 2011. MMWR Morb Mortal Wkly Rep. 2012;61(2):37-8.

5. Centers for Disease Control and Prevention (CDC) Division of Parasitic Diseases. Diagram of the lifecycle of Sarcocystis spp. Atlanta: CDC. [Accessed 27 Oct 2012]. Available from: http:// dpd.cdc.gov/dpdx/html/imagelibrary/s-z/sarcocystosis/body_ sarcocystosis_il5.htm

6. Fayer R. Sarcocystis spp. in human infections. Clin Microbiol Rev. 2004;17(4):894-902.

7. 7.Dubey JP, Speer CA, Fayer R. Sarcocystosis of animals and man. Boca Raton, Florida: CRC Press; 1989.

8. Thomas V, Dissanaike AS. Antibodies to Sarcocystis in Malaysians. Trans R Soc Trop Med Hyg. 1978;72(3):303-6.

9. Wong KT, Pathmanathan R. High prevalence of human skeletal muscle sarcocystosis in south-east Asia. Trans R Soc Trop Med Hyg. 1992;86(6):631-2.

10. Arness MK, Brown JD, Dubey JP, Neafie RC, Granstrom DE. An outbreak of acute eosinophilic myositis attributed to human Sarcocystis parasitism. Am J Trop Med Hyg. 1999;61(4):548-53. 